Review Article

\title{
Circulating Endothelial Microparticles: A Key Hallmark of Atherosclerosis Progression
}

\author{
Keshav Raj Paudel, ${ }^{1}$ Nisha Panth, ${ }^{2}$ and Dong-Wook Kim ${ }^{1}$ \\ ${ }^{1}$ Department of Oriental Medicine Resources, Mokpo National University, Muan-gun, \\ Jeonnam 534-729, Republic of Korea \\ ${ }^{2}$ College of Pharmacy and Natural Medicine Research Institute, Mokpo National University, Muan-gun, \\ Jeonnam 58554, Republic of Korea
}

Correspondence should be addressed to Dong-Wook Kim; mokpou@yahoo.co.kr

Received 30 December 2015; Revised 13 February 2016; Accepted 15 February 2016

Academic Editor: Mark Segal

Copyright ( $) 2016$ Keshav Raj Paudel et al. This is an open access article distributed under the Creative Commons Attribution License, which permits unrestricted use, distribution, and reproduction in any medium, provided the original work is properly cited.

\begin{abstract}
The levels of circulating microparticles (MPs) are raised in various cardiovascular diseases. Their increased level in plasma is regarded as a biomarker of alteration in vascular function. The prominent MPs present in blood are endothelial microparticles (EMPs) described as complex submicron $(0.1$ to $1.0 \mu \mathrm{m})$ vesicles like structure, released in response to endothelium cell activation or apoptosis. EMPs possess both physiological and pathological effects and may promote oxidative stress and vascular inflammation. EMPs release is triggered by inducer like angiotensin II, lipopolysaccharide, and hydrogen peroxide leading to the progression of atherosclerosis. However, there are multiple physiological pathways for EMPs generation like NADPH oxidase derived endothelial ROS formation, Rho kinase pathway, and mitogen-activated protein kinases. Endothelial dysfunction is a key initiating event in atherosclerotic plaque formation. Atheroemboli, resulting from ruptured carotid plaques, is a major cause of stroke. Increasing evidence suggests that EMPs play an important role in the pathogenesis of cardiovascular disease, acting as a marker of damage, either exacerbating disease progression or triggering a repair response. In this regard, it has been suggested that EMPs have the potential to act as biomarkers of disease status. This review aims to provide updated information of EMPs in relation to atherosclerosis pathogenesis.
\end{abstract}

\section{What Are Microparticles?}

Microparticles (MPs) are cell membrane-shedded submicron fragments ranging from 0.1 to $1.0 \mu \mathrm{m}$ containing information like mRNA, microRNAs (miRNAs), receptor, and specific proteins of parent cell. MPs are expressed by cells during cellular stress and cell activation [1]. To date, MPs have been identified as membrane vesicles released from blood-related cells such as endothelial cells, smooth muscle cells, platelets, erythrocytes, and leukocytes, as recognized by their specific surface proteins $[2,3]$. In history, MPs were regarded as "cell dust/debris" and the process of their formation remains to be completely elucidated $[4,5]$. However, it is clear that MPs formation and shedding involve reconstitution of cell membrane phospholipid structure, with outer leaflet subjected to phosphatidylserine (PS), and alteration of the cell architecture, with the disruption of cytoskeleton organization [6-9]. Several studies report the existence of small vesicles similar in size as MPs but do not expose PS in the outer leaflet. The area of MP study is rapidly growing as the role of MPs in the pathogenesis of diabetes mellitus [10], lungs cancer [11, 12], central nervous system disorder [13], inflammation [14, 15], systemic lupus erythematosus [16], and cardiovascular diseases (CVDs) [17] has been highlighted by various clinical and experimental research.

\section{Types of Microparticles}

The types of microparticles and their role in disease progression are summarized in Table 1. 
TABLE 1: The types of microparticles and their role in disease progression.

\begin{tabular}{|c|c|c|}
\hline Microparticles & Role in disease progression & Reference \\
\hline PMP & $\begin{array}{l}\text { Coagulation, inflammatory processes, thrombosis, and tumor } \\
\text { progression }\end{array}$ & [19-21] \\
\hline EMP & $\begin{array}{c}\text { Endothelial dysfunction, angiogenesis, tumor growth, and increased } \\
\text { oxidative stress }\end{array}$ & {$[11,22-24]$} \\
\hline MMP & Endothelial dysfunction, sepsis, and vascular inflammation & {$[25-27]$} \\
\hline
\end{tabular}

\section{Isolation of Microparticles}

Although, some of the research studies on MPs derived ex vivo from blood of suitable patient, but due to the less number of MPs derived from this methods, various in vitro cells lines (e.g., Jurkat cells and human umbilical vein endothelial cells) stimulated with cytokine, chemicals, or apoptosis inducer are use in research laboratory to obtain MPs. Various inducers like angiotensin II, lipopolysaccharide, and hydrogen peroxide can be applied to generate MPs in vitro.

In brief, the MPs containing the suspension of cell, treated with suitable stimulus, are subjected to initial low speed centrifugation at $\sim 1500 \times \mathrm{g}$ to remove the cells followed by second high speed centrifugation at $\sim 20,000 \times$ g to get MPs pellet at the bottom. After removing the supernatant, these MPs are suspended on saline buffer solution, divided as necessary, and stored at $-70-80^{\circ} \mathrm{C}$ until further use. In contrast to this method, some researchers also used MPs pellets after ultracentrifugation at a speed of $100,000 \times \mathrm{g}$. In this case, not only MPs, but also exosomes vesicles of diameter 40-90 nm settle down at the bottom as sediment [18].

\section{What Are Endothelial Microparticles?}

In human blood circulation, endothelial MPs (EMPs) constitute a smaller population of MP family. However, it has been widely associated with pathogenesis of various CVDs, mainly initiated by endothelial dysfunction [22, 45, 46]. Researches have demonstrated the key role of EMPs in the growth, division, and maturation of endothelial precursor cells, important for blood vessel regeneration [47], suggesting a potential preservative role in response to vascular regeneration, restoration, and protection [48]. It is of great interest to know the difference in the level of EMPs in healthy and pathological condition. In this aspect, Mezentsev et al. [49] have carried out experiment to highlight the various designs of angiogenesis (cell proliferation rate, capillary formation, and death of endothelial cells) by in vitro assay, quantifying physiological levels of EMPs in healthy volunteers (ranging from $10^{3}$ to $\left.10^{4} \mathrm{EMPs} / \mathrm{mL}\right)[33,50]$ and diseased state concentrations (measured in person with cardiovascular disease, $10^{5} \mathrm{EMPs} / \mathrm{mL}$ ) [51-53]. Higher levels of EMPs are believed to affect angiogenesis, with the amplitude of the effect being directly proportional to EMPs concentration. Another study by Koga et al. found the presence of cluster of differentiation (CD) 144 also known as vascular endothelial- (VE-) cadherin positive EMPs in human circulation, with higher levels being seen in those suffering from diabetic mellitus and coronary artery disease including atherosclerosis. Consequently, VE-cadherin positive EMPs can be a hallmark for analyzing atherosclerosis via endothelial cell dysfunction. Taken together, various cardiovascular complications can be efficiently prevented by therapeutics approach that target atherosclerosis patients focusing on assessments of blood CD144-EMP levels [34].

\section{What Is Atherosclerosis?}

Atherosclerosis is described as a vascular inflammatory disorder characterized by narrowing of blood vessel lumen due to accumulation of lipid, inflammatory cell, vascular smooth muscle cell (VSMC), and platelet triggered by lipid peroxidation, endothelial dysfunction, release of inflammatory mediators by activated macrophage, and VSMC migration and proliferation in intimal layer $[54,55]$. It is initiated by endothelial dysfunction whereby endothelial cells produce chemoattractant factors that induce monocyte recruitment and infiltration in the neointima. At intimal site, monocytes differentiate into macrophages and engulf oxidized lowdensity lipoproteins (LDLs) derived from the circulation to the site of lesion, leading to the formation of inflammatory foam cells. Atherosclerotic lesion progresses with exacerbated macrophage accumulation and release of various inflammatory mediators such as cyclooxygenase-2 (COX-2), inducible nitric oxide synthase (iNOS), tumor necrosis factor (TNF- $\alpha$ ), and interleukin followed by VSMC migration and proliferation from the media to the intima due to the activation of mitogen-activated protein kinases (MAPKs) pathways, collagen production, and lesion calcification $[56,57]$. Atheroma buildup is facilitated by platelet and other circulating cell accumulation. Eventually, the brust of the atherosclerotic plaque due to high blood pressure results in the occlusion of artery lumen mainly in small artery with narrow lumen and further complication. Atherosclerosis develops quietly many years ago before clinical manifestation is revealed. However, early identification of atheroma formation may allow measures to prevent the progression of the acute condition towards serious clinical events (Figure 1). Since imaging techniques do not allow early detection and cannot be used routinely, finding circulating biomarkers is of great importance to predict cardiovascular disease risk and pathology prognosis [58, 59]. 


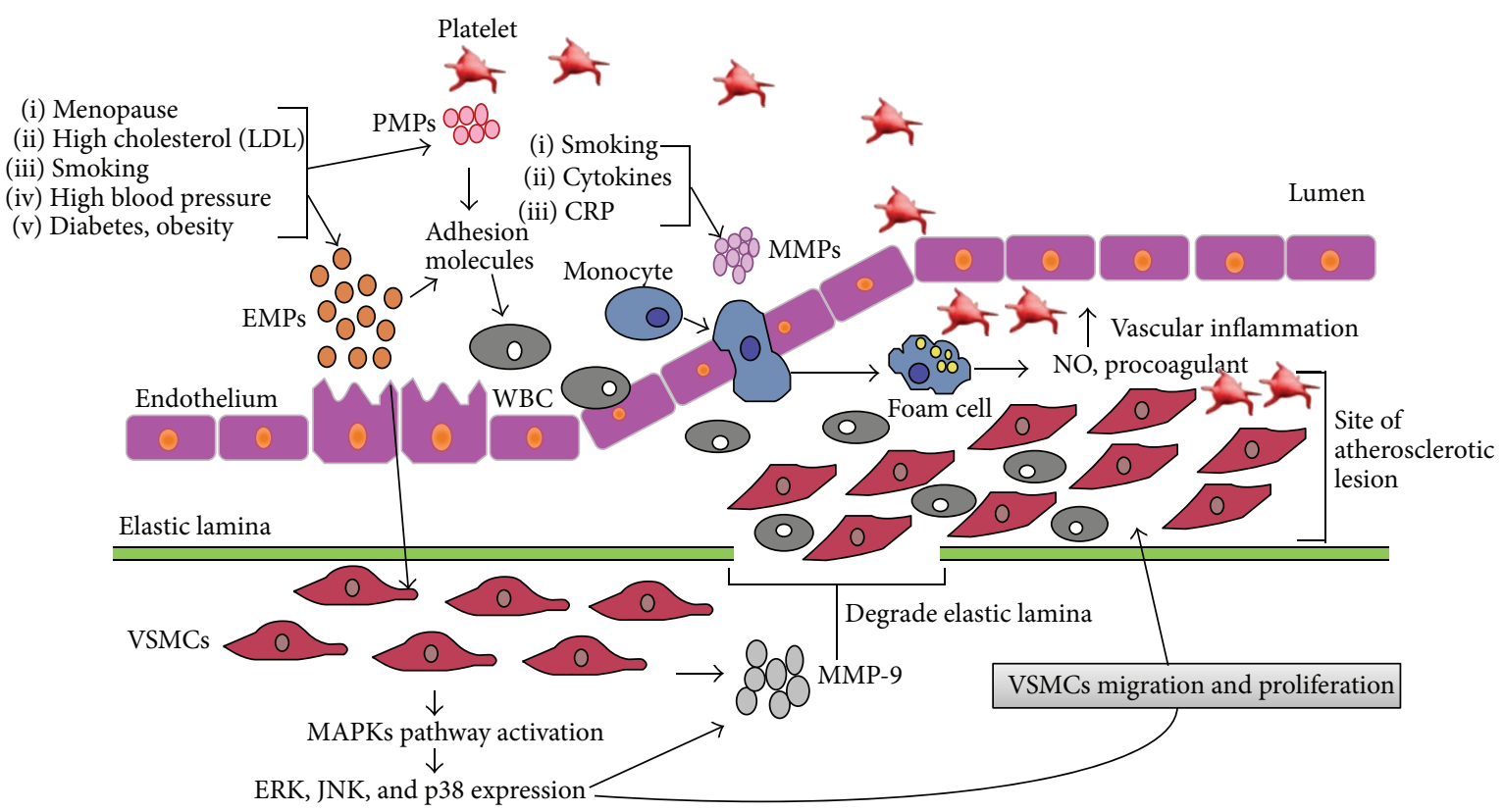

Figure 1: The chain of events initiated by MPs on atherosclerosis physiopathology. Endothelial microparticles (EMPs) and platelet microparticles (PMPs) are generated by the activation of endothelium with a number of factors like smoking, oxidative stress, obesity, and high blood pressure whereas monocyte-microparticles (MMPs) are generated by proinflammatory cytokines (tumor necrosis factor$\alpha$, interleukin 1- $\beta$ ), cigarette smoking, and C-reactive protein (CRP). EMPs can activate vascular smooth muscle cells (VSMCs) to express matrix metalloproteinase-9 (MMP-9) that can degrade elastic lamina barrier to facilitate VSMC migration from tunic media to tunic intima at the site of atherosclerotic lesion. Furthermore, EMPs also upregulate ERK, JNK, and p38 through MAPKs pathway leading to proliferation and migration of VSMC to develop atheroma plaque. Cell adhesion molecules expressed by EMPs and PMPs facilitate leukocytes and platelets adhesion to the endothelial surface thereby developing intimal thickening and vascular lesion. MMPs can activate inducible nitric oxide synthase pathway to release nitric oxide (NO) and procoagulant responsible for vascular inflammation and endothelial dysfunction. Collectively, all this event leads to atherosclerosis.

\section{Role of EMPs on Pathogenesis of Atherosclerosis}

The scientific highlight about the role of MPs on atherosclerosis progression is summarized in Table 2.

6.1. Association of Oxidative Stress with EMPs. The role of oxidative stress as a stimulus for EMPs release facilitating atherosclerosis progression was described by Szotowski et al. and Vince et al. According to the study done by Szotowski et al., the evidence of the relationship among reactive oxygen spices (ROS) formation and production of EMP-associated tissue factor was established. Furthermore, the pretreatment of any antioxidant compound before application of ionization radiation (source of ROS formation) and TNF- $\alpha$ induction to endothelial cell remarkably inhibited ROS formation. This inhibition of ROS production was accompanied with a lower expression of thrombogenic EMPs, highlighting the positive relationship of ROS and the formation of thrombogenic EMPs [35]. When cells are lacking enough oxygen, they are metabolically impaired resulting in compromised cellular function eventually leading to apoptosis via metabolic failure. Apoptosis is a potent signal for EMPs generation [1]. Polymorphoneutrophils (PMNs) bound to the endothelial surface upregulate endothelial dysfunction and histological damage through a variety of noxious pathways. At this point, activated neutrophils moderate a variety of deleterious responses like generation of ROS, cytotoxic enzymes, and inflammation mediators or cytokines. Moreover, they also hire additional neutrophils by chemotaxis and enhance their attachment to the vascular intimal endothelium though adhesion glycoprotein molecules were expressed on both the surfaces of the neutrophil and endothelial cell. High levels of ROS can distort the redox equilibrium of cells causing oxidative stress and destruction to cell membrane organization followed by membrane microparticle release, protein degradation, and apoptosis [15].

6.2. Monocyte-Macrophage MPs in Atherosclerosis. In patients with atherosclerosis, inflammatory macrophage pools are enlarged in the atherosclerotic plaques. Monocytemacrophage-derived MPs are critical part of the progression of unstable atherosclerotic plaques. Monocyte-macrophages express MPs on activation by cigarette consumption, proinflammatory cytokines (TNF- $\alpha$, IL-1 $\beta$ ) and C-reactive protein. Apart from this, endotoxin induces macrophage MPs formation via a pathway essential for inducible nitric oxide synthase (iNOS) activation [40]. It is well established that iNOS overexpression greatly influence vascular inflammation by the generation of significant amount of nitic oxide (NO) [54]. Furthermore, tobacco smoke provokes the generation of highly procoagulant monocytic MPs in a process requiring 
TABLE 2: Scientific work on microparticle in association with its link with atherosclerosis.

\begin{tabular}{|c|c|c|}
\hline Microparticles & Role in atherosclerosis & Reference \\
\hline \multirow{5}{*}{ PMPs } & $\begin{array}{l}\text { Enhance cyclooxygenase-2 (COX-2) and intracellular adhesion molecules-1 } \\
\text { (ICAM-1) release. }\end{array}$ & {$[28]$} \\
\hline & $\begin{array}{l}\text { COX-2 expression in monocytes through translocation of protein kinase } \mathrm{C}(\mathrm{PKC}) \\
\text { from cytosol to the cell membrane. }\end{array}$ & [29] \\
\hline & Enhance the adhesion of monocyte to human umbilical vein endothelial cells. & [30] \\
\hline & Increase WBC aggregation and assembly via expression of P-selectin. & [31] \\
\hline & Neutrophil aggregation and enhance phagocytic activity. & [32] \\
\hline \multirow{6}{*}{ EMPs } & CD-144/vascular endothelial cadherin positive. & {$[33]$} \\
\hline & Reactive oxygen spices (ROS) formation. & {$[34]$} \\
\hline & Matrix metalloproteinase-2 (MMP-2) activation and vascular matrix remodeling. & {$[35,36]$} \\
\hline & $\begin{array}{l}\text { Reduce expression of p38-MAPK in human aortic endothelial cells caused } \\
\text { reduction of TNF- } \alpha \text { induced EMPs. }\end{array}$ & {$[37]$} \\
\hline & EMPs exhibit the TF activity in atherosclerotic plaques. & [38] \\
\hline & Expression of E-selectin, ICAM-1, and VCAM-1. & [39] \\
\hline \multirow{7}{*}{ MMPs } & Inducible nitric oxide synthase (iNOS) activation. & [40] \\
\hline & Extracellular signal-regulated kinases (ERK1/2) activation. & [41] \\
\hline & Procoagulant substances responsible for endothelial dysfunction. & [25] \\
\hline & IL-1 $\beta$ in MMPs can enhance the vascular inflammatory. & {$[42]$} \\
\hline & Atherosclerotic plaque formation in the $\mathrm{ApoE}^{-/-}$mice. & {$[27]$} \\
\hline & $\begin{array}{l}\text { Increased expression of apoptosis regulator Bax, caspase- } 8 \text {, caspase-3, and } \\
\text { cytochrome } \mathrm{C} \text { in cardiomyocytes. }\end{array}$ & {$[43]$} \\
\hline & $\begin{array}{l}\text { Induce superoxide anion production, inflammatory cytokine release, and activation } \\
\text { of NF- } \kappa \text { B gene in monocytes. }\end{array}$ & {$[44]$} \\
\hline
\end{tabular}

extracellular signal-regulated kinases (ERK1/ERK2) activation [41]. ERK activation is a factor causing VSMC proliferation in atherosclerosis [55]. The MPs originated from monocytes contain procoagulant substances responsible for endothelial dysfunction [25]. A research done by Wang et al. showed that the presence of IL-1 $\beta$ in monocytemacrophage MPs can enhance the vascular inflammatory process and activate endothelial cells [42]. Similarly, Hoyer et al. demonstrated the role of monocyte-macrophage MPs in atherosclerotic vascular inflammation and showed that the application of monocyte-macrophage MPs in $\mathrm{ApoE}^{-/-}$ mice facilitated the development of atherosclerotic plaque in the gene knock-out mice and also increase the buildup of macrophages in the vessel wall. This study has clarified crucial interaction between monocyte-macrophage EMPs and vascular inflammatory in the atherosclerotic disease progression of ApoE ${ }^{-/-}$mice [27]. Human atherosclerotic plaques contain MPs released from various apoptotic cell deaths within the necrotic lesion site. Milbank et al. investigated the role of MPs generated by apoptotic murine macrophage (RAW 264.7) cell in adult murine cardiomyocytes. The results showed that MPs contained the soluble form of TNF- $\alpha$. After application of MPs, there was increased expression of apoptosis regulator bcl-2-like protein 4 (Bax), caspase-8, caspase-3, and cytochrome $\mathrm{C}$ in cardiomyocytes. These results provide evidence that MPs released from activated macrophages possessing TNF- $\alpha$ could contribute to the promotion of vascular inflammatory signals leading to atherosclerosis and myocardial infraction [43].
6.3. EMPs and Matrix Metalloproteinase Association in Atherosclerosis. Considerable research favours that microparticles could induce expression of metalloproteinases (MMPs), thereby degrading extracellular matrix (ECM) component by its proteolytic activity $[60,61]$. EMPs play an important role in plasmin (enzyme needed to activate MMPs) localization and activation. MMP-2 and MMP-9 functions are affected by MPs produced by cell other than endothelial cells $[60,62]$. For atherosclerosis event, MMP-9 and MMP2 play key role by degrading ECM and facilitating VSMC migration from media to intima of blood vessel [54]. Lozito and Tuan disclose the endogenous MMPs' proteolytic activity of EMPs by expressing both membrane-type MMPs and soluble molecules believed to be crucial in maintaining ECM. EMPs can activate endogenous zymogen, proMMP-2, a feature not seen in the remaining endothelial cell secreted factors to provide the evidence of MP-induction of MMP-2 with MMP-14. Endothelial cell secreted factors include the increasing concentration of tissue inhibitors of metalloproteinase-1 (TIMP-1) and TIMP-2, which were not detected in MPs [36]. The release of ECM cleaving proteases, particularly MMPs by endothelial cells, is an important process during angiogenesis and atherosclerosis. Taraboletti et al. found that in vitro cultured human umbilical vein endothelial cells (HUVEC) shed microparticles in the range of $0.3-0.6 \mu \mathrm{m}$ from the cells plasma membrane, as observed by ultrastructural analysis. Detailed analysis of microparticles showed that the two principle gelatinases essential for atherosclerosis progression, MMP-2 and MMP-9, were discovered on the outer side of 
the microparticles membrane. It is clear that endothelial cells upon stimulation by various means shed MMP-containing microparticles and this is responsible for regulating proteolytic activity of MMPs to degrade the ECM and promote VSMC migration for atheroma formation in neointima [60].

6.4. EMPs Linked with MAPKs Signaling Pathway. MAPK is a cell signaling pathway directly related to atherosclerosis pathophysiology as it induces proliferation and migration of vascular smooth muscle cell $[53,55]$. MAPK is a family of three proteins, namely, extracellular signal-regulated kinases (ERK), c-jun terminal $\mathrm{NH}_{2}$-kinase (JNK), and p38 [56]. Previous researches have demonstrated the activation of different cell signaling pathways during EMPs release. In vitro experiment has shown that proinflammatory mediators can cause endothelial apoptosis and activation, thereby increasing EMPs basal release through MAPKs dependent pathways [48]. p38-MAPK pathways are involved in controlling the TNF- $\alpha$ dependent EMPs release [37, 63]. It was observed that reducing the expression of p38-MAPK in human aortic endothelial cells, using therapeutics agents, caused the reduction of TNF- $\alpha$-induced EMPs by the half. It is interesting to note that the suppression of MPs was focused on p38 MAPK pathways. These findings revealed p38 MAPK signaling as potent and specific in the generation and maturation of EMPs and as an unidirectional model where p38 MAPK serves as critical source of microparticle formation, unlike the target cellular response to EMPs [37]. Likewise, it also proposes a new pathway which offer in vivo therapeutic benefit via direct inhibition of EMP formation by p38 inhibition. [37]. In physiological system, during the initial events of atherosclerosis progression, intense tissue factor (TF) generation is seen in monocytes. However, at later advance stages of atherosclerosis, TF release is also observed in modified macrophage (foam cells), endothelial cells, and VSMC [64]. $\mathrm{TF}$ exists in the necrotic lesion of atheroma plaques as well, particularly linked with MPs derived from apoptotic foam cells, macrophages, or lymphocytes. These MPs of course exhibit the TF activity in atherosclerotic plaques [38]. TF bearing microparticles are released by endothelial cell induced by cytokines such as TNF- $\alpha$, IL-1 $\beta,[65]$ and the MAPKs p38, ERK, and JNK are involved in TNF- $\alpha$-induced TF expression [66].

6.5. EMPs Induced Vascular Inflammation. The influence of MPs on vascular inflammation to cause atherosclerosis has been studied in detail. Considerable studies favour a role for MPs in upregulating proinflammatory mediators like IL6 , monocyte chemoattractant protein-1 (MCP-1), inducible nitric oxide synthase (iNOS), and cyclooxygenase-2 (COX2) release by endothelial cells following JNK1 and nuclear factor kappaB (NF- $\kappa \mathrm{B})$ pathways $[67,68]$ and TNF- $\alpha$ and IL-1 by monocytes [69]. Arachidonic acid, a marker of cell injury/inflammation, secreted from platelet-derived MPs, enhances COX-2 and intracellular adhesion molecules-1 (ICAM-1) release in endothelial cells as well as COX-2 expression in monocytes through the translocation of protein kinase $\mathrm{C}$ (PKC) from the cytosol to the cell membrane [28, 29]. Furthermore, aminophospholipids placed on the MPs membrane can also boost inflammation. Aminophospholipids act as substrates for phospholipase A2, responsible for catalyzing the production of lysophosphatidic acid (LPA), a factor of vascular inflammation [70]. EMPs release is also induced by thrombin mediated inflammatory particles like IL-8 and interleukin-1 receptor antagonists (IL-1Ra) and IL-1 that are involved in blood coagulation, vascular inflammation, and angiogenesis [71, 72]. Vascular inflammation and coagulation are linked processes as they collectively build up atheroma in blood vessel wall, and, in this step, MPs may intensify the responses by activating NF- $\kappa \mathrm{B}$, elevating nitric oxide (NO) release, and increasing oxidative stress $[9,73]$. Scientific evidence evinces that MPs induce harmful effects on vascular function via the release of inflammatory cytokines/ chemokines as well as increased expression of endothelial cell adhesion molecules [29, 30, 74]. MPs obtained from white blood cells (WBC) also circulate along with blood at low levels in individuals with good health and are at high levels during vascular inflammation [67]. In vitro experiment revealed that such MPs can activate endothelial cells leading to gene expression and liberate inflammatory mediators (IL6 and IL-8) and upregulation of WBC-endothelial ICAM1, vascular adhesion molecule-1 (VCAM-1), and E-selectin [74]. Likewise, MPs released following platelet activation may magnify WBC aggregation and assembly via the expression of P-selectin, adding further inflammation and buildup of atheroma plaque [31]. Similarly, platelet-derived MPs bind to neutrophils to cause neutrophil aggregation and enhance phagocytic activity, a common event seen after vascular inflammation [32]. Recently, it was revealed that monocyteacquired MPs have exhibited the induction of oxidative stress marker (superoxide anion production), inflammatory cytokine release, and activation of NF- $\kappa \mathrm{B}$ gene in monocytes [44]. Taken together, these results demonstrate that, in atherosclerosis, increased levels of MPs may intensify inflammation and vascular injury.

6.6. EMPs Induced Cell Adhesion Molecules. It is well established that EMPs carry many surface molecules from the origin cell to show physiological function. A common such molecule is cell adhesion molecule which includes platelet endothelial cell adhesion molecule-1 (PECAM-1), VCAM-1, and ICAM-1 [47]. A crucial event of atheroma formation involves the adhesion of circulating monocytes on the vascular endothelium mediated by these cell adhesion molecules. A study showed that MPs and exosomes from platelets collectively enhance endothelial cell ICAM-1 and the adhesion of monocytic U937 cells to human umbilical vein endothelial cells [30]. Based on the type of stimulus for endothelial cell injury, either apoptosis or activation, the surface antigens molecules of EMPs are varied. Jimenez et al. investigated that increased amounts of the surface antigens E-selectin, ICAM1 , and VCAM-1 are present on EMPs obtained from induced endothelial cells, whereas in the case of EMPs derived from apoptotic endothelial cells less amounts of those surface antigens were present. Also, VE-cadherin, PECAM-1, and endoglin are at minimum levels on EMPs acquired from induced ECs $[10,39,75,76]$. MPs derived from in vitro assay also induce cell adhesion molecules expression, specifically 
ICAM-1, at the surface of endothelial cells [30, 37, 77]. Likewise in in vivo human study, MPs isolated from human atherosclerotic plaques enhance endothelial cell expression of ICAM-1 following monocytes attachment to the intimal layer, but MPs derived from plasma possess no such function. The mechanism of enlarged endothelial ICAM-1 prompt by atherosclerotic plaque EMPs follows the conveyance of this cell adhesion molecule in a phosphatidylserine-dependent pathway from MPs to endothelial cells [78]. The role of atherosclerotic plaque MPs on the recruitment of inflammatory cells mediated by ICAM-1 might be distinctly applicable at the level of blood vessel ongoing advanced atherosclerotic lesions, since neointimal highly express VCAMs in comparison to luminal arterial endothelium [79]. These unusual neovessels are featured by random division of vessel branch having immature endothelial lining with altered permeability $[80,81]$. For this reason, atherosclerotic plaques MPs bearing ICAM-1 may disperse along with blood flow and hence carry ICAM-1 to the surface of endothelial cell in "paracrine" manner [78].

\section{Clinical Relevance of EMPs on Disease Related to Atherosclerosis}

A number of diseases or physiological conditions like obesity and menopause associated with circulating MPs are also related to atherosclerosis progression. In menopausal women, the decrease level of endogenous estrogen possesses risk for vascular inflammation as the normal level of endogenous estrogen helps to maintain the anti-inflammatory, anticoagulant, and antithrombotic potency of endothelium. The mechanism behind this include balancing NO production, lowering oxidative stress, and decreasing the expression of procoagulant cell adhesion molecule [82]. High level of cellular MPs and proinflammatory cytokine in postmenopausal women leads to alterations of these cell adhesion molecules facilitating leukocytes and platelets adhesion to the endothelial surface thereby developing intimal thickening and vascular lesion [83]. So, it is likely that women at menopause are going through high risk of metabolic syndrome (MS) responsible for cardiovascular disease. Muthuvel Jayachandran et al. hypothesized that the changes in platelet function and levels of MPs obtained from blood and vascular cell during early menopause may reveal a link to the early atherothrombotic progression and found that, among recently menopausal women, specific platelet functions and level of cell membrane-derived procoagulant MPs alter individual components of MS. These alterations in cellular level clearly highlight how menopause contributes to MS and finally cardiovascular disease including atherosclerosis [84].

Obesity and type 2 diabetes patients are at increased risks of atherosclerosis and other cardiovascular complications since MPs are raised in blood plasma of diabetic, obese, and insulin-resistant individuals. An in vivo study done in 74 patients undergoing total knee arthroplasty reveals that the level of EMPs was higher in obese patient as compared to nonobese one. The level of EMPs was raised promptly after surgery, while 3 days after operation EMPs were restored to the basal level as before operation except in the case of obese patients. Furthermore, endothelial progenitor cells were less in obese patients, whereas the level of adipokines was raised after surgery. This suggests a vascular inflammatory condition that exacerbates adipokines level after the operation and may worsen endothelial repair [85]. An animal model to study the various pathways followed by MPs to promote atherosclerosis by augmenting endothelial dysfunction and upregulating release of proinflammatory mediators is highfat diet (HFD) fed rats for prolonged period to make them obese and insulin resistant. Heinrich et al. [86] found that total MPs were drastically raised after feeding rats with HFD compared to normal basal diet. In this study, significantly high levels of MPs were obtained from endothelial, leucocyte, and platelet. MPs isolated from HFD fed rats possess the ability to stimulate ROS generation as well as expression of VCAM-1, an indication of a proinflammatory nature of MPs in this model.

\section{Conclusions}

Endothelial microparticle release is triggered by various stimulations followed by the activation of various pathways that can collectively make progress in the atherogenesis. Most importantly, oxidative stress, endogenous molecules (TNF- $\alpha$, PDGF, and interleukin), and apoptosis inducer are responsible for EMPs release to undergo functional change in blood vessel eventually leading to the pathology of atherosclerosis. It is worth noting that various physiological and pathological conditions like menopause, obesity, and diabetic can promote atherosclerosis by generation of MPs. Furthermore, monocyte-macrophage-derived MPs are also critical part of the progression of unstable atherosclerotic plaques. Hence, medicine targeting the downregulation of EMPs as well as other MPs release can be a possible therapeutic option treating vascular inflammatory disease like atherosclerosis.

\section{Competing Interests}

The authors declare that they have no competing interests.

\section{Authors' Contributions}

All the authors contributed equally to this review.

\section{References}

[1] M. J. VanWijk, E. VanBavel, A. Sturk, and R. Nieuwland, "Microparticles in cardiovascular diseases," Cardiovascular Research, vol. 59, no. 2, pp. 277-287, 2003.

[2] C. N. Franca, M. C. de Oliveira Izar, J. B. do Amaral, D. M. Tegani, and F. A. H. Fonseca, "Microparticles as potential biomarkers of cardiovascular disease," Arquivos Brasileiros de Cardiologia, vol. 104, no. 2, pp. 169-174, 2015.

[3] A. Angelillo-Scherrer, "Leukocyte-derived microparticles in vascular homeostasis," Circulation Research, vol. 110, no. 2, pp. 356-369, 2012.

[4] S. Patz, C. Trattnig, G. Grünbacher et al., "More than cell dust: microparticles isolated from cerebrospinal fluid of brain injured 
patients are messengers carrying mRNAs, miRNAs, and proteins," Journal of Neurotrauma, vol. 30, no. 14, pp. 1232-1242, 2013.

[5] G. Pasterkamp and D. de Kleijn, "Microparticles, debris that hurts," Journal of the American College of Cardiology, vol. 52, no. 16, pp. 1312-1313, 2008.

[6] D. E. Connor, T. Exner, D. D. F. Ma, and J. E. Joseph, "The majority of circulating platelet-derived microparticles fail to bind annexin V, lack phospholipid-dependent procoagulant activity and demonstrate greater expression of glycoprotein Ib," Thrombosis and Haemostasis, vol. 103, no. 5, pp. 1044-1052, 2010.

[7] M. Sekuła, G. Janawa, E. Stankiewicz, and E. Stepień, "Endothelial microparticle formation in moderate concentrations of homocysteine and methionine in vitro," Cellular and Molecular Biology Letters, vol. 16, no. 1, pp. 69-78, 2011.

[8] M. G. Macey, N. Enniks, and S. Bevan, "Flow cytometric analysis of microparticle phenotype and their role in thrombin generation," Cytometry Part B: Clinical Cytometry, vol. 80, no. 1, pp. 57-63, 2011.

[9] N. Amabile, A. P. Guérin, A. Leroyer et al., "Circulating endothelial microparticles are associated with vascular dysfunction in patients with end-stage renal failure," Journal of the American Society of Nephrology, vol. 16, no. 11, pp. 3381-3388, 2005.

[10] A. F. Tramontano, R. Lyubarova, J. Tsiakos, T. Palaia, J. R. Deleon, and L. Ragolia, "Circulating endothelial microparticles in diabetes mellitus," Mediators of Inflammation, vol. 2010, Article ID 250476, 8 pages, 2010.

[11] C.-C. Wang, C.-C. Tseng, C.-C. Hsiao et al., "Circulating endothelial-derived activated microparticle: a useful biomarker for predicting one-year mortality in patients with advanced non-small cell lung cancer," BioMed Research International, vol. 2014, Article ID 173401, 11 pages, 2014.

[12] C.-C. Tseng, C.-C. Wang, H.-C. Chang et al., "Levels of circulating microparticles in lung cancer patients and possible prognostic value," Disease Markers, vol. 35, no. 5, pp. 301-310, 2013.

[13] S. M. Schindler, J. P. Little, and A. Klegeris, "Microparticles: a new perspective in central nervous system disorders," BioMed Research International, vol. 2014, Article ID 756327, 17 pages, 2014.

[14] D. M. Babbitt, K. M. Diaz, D. L. Feairheller et al., "Endothelial activation microparticles and inflammation status improve with exercise training in African Americans," International Journal of Hypertension, vol. 2013, Article ID 538017, 8 pages, 2013.

[15] R. V. Vince, B. Chrismas, A. W. Midgley, L. R. McNaughton, and L. A. Madden, "Hypoxia mediated release of endothelial microparticles and increased association of S100A12 with circulating neutrophils," Oxidative Medicine and Cellular Longevity, vol. 2, no. 1, pp. 2-6, 2009.

[16] J. Perez-Hernandez and R. Cortes, "Extracellular vesicles as biomarkers of systemic lupus erythematosus," Disease Markers, vol. 2015, Article ID 613536, 7 pages, 2015.

[17] A. Celi, R. Lorenzet, B. C. Furie, and B. Furie, "Microparticles and a P-selectin-mediated pathway of blood coagulation," Disease Markers, vol. 20, no. 6, pp. 347-352, 2004.

[18] S. F. Mause and C. Weber, "Microparticles: protagonists of a novel communication network for intercellular information exchange," Circulation Research, vol. 107, no. 9, pp. 1047-1057, 2010.

[19] B. Toth, S. Liebhardt, K. Steinig et al., "Platelet-derived microparticles and coagulation activation in breast cancer patients," Thrombosis and Haemostasis, vol. 100, no. 4, pp. 663669,2008 .

[20] M. S. Williams, H. L. Rogers, N.-Y. Wang, and R. C. Ziegelstein, "Do platelet-derived microparticles play a role in depression, inflammation, and acute coronary syndrome?" Psychosomatics, vol. 55, no. 3, pp. 252-260, 2014.

[21] S. Mezouar, D. Mege, R. Darbousset et al., "Involvement of platelet-derived microparticles in tumor progression and thrombosis," Seminars in Oncology, vol. 41, no. 3, pp. 346-358, 2014.

[22] V. Faure, L. Dou, F. Sabatier et al., "Elevation of circulating endothelial microparticles in patients with chronic renal failure," Journal of Thrombosis and Haemostasis, vol. 4, no. 3, pp. 566-573, 2006.

[23] S. Tual-Chalot, F. Gagnadoux, W. Trzepizur, P. Priou, R. Andriantsitohaina, and M. C. Martinez, "Circulating microparticles from obstructive sleep apnea syndrome patients induce endothelin-mediated angiogenesis," Biochimica et Biophysica Acta, vol. 1842, no. 2, pp. 202-207, 2014.

[24] F. Cheng, Y. Wang, J. Li et al., "Berberine improves endothelial function by reducing endothelial microparticles-mediated oxidative stress in humans," International Journal of Cardiology, vol. 167, no. 3, pp. 936-942, 2013.

[25] B. Wen, V. Combes, A. Bonhoure, B. B. Weksler, P.-O. Couraud, and G. E. R. Grau, "Endotoxin-induced monocytic microparticles have contrasting effects on endothelial inflammatory responses," PLoS ONE, vol. 9, no. 3, Article ID e91597, 2014.

[26] R. Nieuwland, R. J. Berckmans, S. McGregor et al., "Cellular origin and procoagulant properties of microparticles in meningococcal sepsis," Blood, vol. 95, no. 3, pp. 930-935, 2000.

[27] F. F. Hoyer, M. K. Giesen, C. Nunes França, D. Lütjohann, G. Nickenig, and N. Werner, "Monocytic microparticles promote atherogenesis by modulating inflammatory cells in mice," Journal of Cellular and Molecular Medicine, vol. 16, no. 11, pp. 27772788, 2012.

[28] O. P. Barry, M. G. Kazanietz, D. Praticò, and G. A. FitzGerald, "Arachidonic acid in platelet microparticles up-regulates cyclooxygenase- 2-dependent prostaglandin formation via a protein kinase $\mathrm{C} /$ mitogen-activated protein kinase-dependent pathway," Journal of Biological Chemistry, vol. 274, no. 11, pp. 7545-7556, 1999.

[29] O. P. Barry, D. Praticò, J. A. Lawson, and G. A. FitzGerald, "Transcellular activation of platelets and endothelial cells by bioactive lipids in platelet microparticles," Journal of Clinical Investigation, vol. 99, no. 9, pp. 2118-2127, 1997.

[30] O. P. Barry, D. Praticò, R. C. Savani, and G. A. FitzGerald, "Modulation of monocyte-endothelial cell interactions by platelet microparticles," Journal of Clinical Investigation, vol. 102, no. 1, pp. 136-144, 1998.

[31] S. B. Forlow, R. P. McEver, and M. U. Nollert, "Leukocyteleukocyte interactions mediated by platelet microparticles under flow," Blood, vol. 95, no. 4, pp. 1317-1323, 2000.

[32] W. Jy, W.-W. Mao, L. L. Horstman, J. Tao, and Y. S. Ahn, "Platelet microparticles bind, activate and aggregate neutrophils in vitro," Blood Cells, Molecules, and Diseases, vol. 21, no. 3, pp. 217-231, 1995.

[33] J. J. Jimenez, W. Jy, L. M. Mauro, L. L. Horstman, C. Soderland, and Y. S. Ahn, "Endothelial microparticles released in thrombotic thrombocytopenic purpura express von Willebrand factor and markers of endothelial activation," British Journal of Haematology, vol. 123, no. 5, pp. 896-902, 2003. 
[34] H. Koga, S. Sugiyama, K. Kugiyama et al., "Elevated levels of VEcadherin-positive endothelial microparticles in patients with type 2 diabetes mellitus and coronary artery disease," Journal of the American College of Cardiology, vol. 45, no. 10, pp. 16221630, 2005.

[35] B. Szotowski, S. Antoniak, P. Goldin-Lang et al., "Antioxidative treatment inhibits the release of thrombogenic tissue factor from irradiation- and cytokine-induced endothelial cells," Cardiovascular Research, vol. 73, no. 4, pp. 806-812, 2007.

[36] T. P. Lozito and R. S. Tuan, "Endothelial cell microparticles act as centers of matrix metalloproteinsase-2 (MMP-2) activation and vascular matrix remodeling," Journal of Cellular Physiology, vol. 227, no. 2, pp. 534-549, 2012.

[37] A. M. Curtis, P. F. Wilkinson, M. Gui, T. L. Gales, E. Hu, and J. M. Edelberg, "p38 mitogen-activated protein kinase targets the production of proinflammatory endothelial microparticles," Journal of Thrombosis and Haemostasis, vol. 7, no. 4, pp. 701-709, 2009.

[38] Z. Mallat, H. Benamer, B. Hugel et al., "Elevated levels of shed membrane microparticles with procoagulant potential in the peripheral circulating blood of patients with acute coronary syndromes," Circulation, vol. 101, no. 8, pp. 841-843, 2000.

[39] J. J. Jimenez, W. Jy, L. M. Mauro, C. Soderland, L. L. Horstman, and Y. S. Ahn, "Endothelial cells release phenotypically and quantitatively distinct microparticles in activation and apoptosis," Thrombosis Research, vol. 109, no. 4, pp. 175-180, 2003.

[40] J. Gauley and D. S. Pisetsky, "The release of microparticles by RAW 264.7 macrophage cells stimulated with TLR ligands," Journal of Leukocyte Biology, vol. 87, no. 6, pp. 1115-1123, 2010.

[41] M. Li, D. Yu, K. Jon Williams, and M.-L. Liu, “Tobacco smoke induces the generation of procoagulant microvesicles from human monocytes/macrophages," Arteriosclerosis, Thrombosis, and Vascular Biology, vol. 30, no. 9, pp. 1818-1824, 2010.

[42] J.-G. Wang, J. C. Williams, B. K. Davis et al., "Monocytic microparticles activate endothelial cells in an IL- $1 \beta$-dependent manner," Blood, vol. 118, no. 8, pp. 2366-2374, 2011.

[43] E. Milbank, R. Soleti, E. Martinez et al., "Microparticles from apoptotic RAW 264.7 macrophage cells carry tumour necrosis factor- $\alpha$ functionally active on cardiomyocytes from adult mice," Journal of Extracellular Vesicles, vol. 4, article 28621, 2015.

[44] C. Bardelli, A. Amoruso, D. Federici Canova et al., "Autocrine activation of human monocyte/macrophages by monocytederived microparticles and modulation by PPAR $\gamma$ ligands," British Journal of Pharmacology, vol. 165, no. 3, pp. 716-728, 2012.

[45] C. M. Boulanger, A. Scoazec, T. Ebrahimian et al., "Circulating microparticles from patients with myocardial infarction cause endothelial dysfunction," Circulation, vol. 104, no. 22, pp. 26492652, 2001.

[46] Z. Mallat, B. Hugel, J. Ohan, G. Lesèche, J.-M. Freyssinet, and A. Tedgui, "Shed membrane microparticles with procoagulant potential in human atherosclerotic plaques: a role for apoptosis in plaque thrombogenicity," Circulation, vol. 99, no. 3, pp. 348353, 1999.

[47] M. Hristov, W. Erl, S. Linder, and P. C. Weber, "Apoptotic bodies from endothelial cells enhance the number and initiate the differentiation of human endothelial progenitor cells in vitro," Blood, vol. 104, no. 9, pp. 2761-2766, 2004.

[48] F. Dignat-George and C. M. Boulanger, "The many faces of endothelial microparticles," Arteriosclerosis, Thrombosis, and Vascular Biology, vol. 31, no. 1, pp. 27-33, 2011.
[49] A. Mezentsev, R. M. H. Merks, E. O’Riordan et al., "Endothelial microparticles affect angiogenesis in vitro: role of oxidative stress," American Journal of Physiology-Heart and Circulatory Physiology, vol. 289, no. 3, pp. H1106-H1114, 2005.

[50] F. Bretelle, F. Sabatier, D. Desprez et al., "Circulating microparticles: a marker of procoagulant state in normal pregnancy and pregnancy complicated by preeclampsia or intrauterine growth restriction," Thrombosis and Haemostasis, vol. 89, no. 3, pp. 486492, 2003.

[51] R. A. Preston, W. Jy, J. J. Jimenez et al., "Effects of severe hypertension on endothelial and platelet microparticles," Hypertension, vol. 41, no. 2, pp. 211-217, 2003.

[52] M. J. VanWijk, K. Boer, R. J. Berckmans et al., "Enhanced coagulation activation in preeclampsia: the role of APC resistance, microparticles and other plasma constituents," Thrombosis and Haemostasis, vol. 88, no. 3, pp. 415-420, 2002.

[53] M. J. VanWijk, R. Nieuwland, K. Boer, J. A. M. Van Der Post, E. VanBavel, and A. Sturk, "Microparticle subpopulations are increased in preeclampsia: possible involvement in vascular dysfunction?" American Journal of Obstetrics \& Gynecology, vol. 187, no. 2, pp. 450-456, 2002.

[54] H.-H. Lee, K. R. Paudel, and D.-W. Kim, "Terminalia chebula fructus inhibits migration and proliferation of vascular smooth muscle cells and production of inflammatory mediators in RAW 264.7,' Evidence-Based Complementary and Alternative Medicine, vol. 2015, Article ID 502182, 10 pages, 2015.

[55] R. Karki, S.-B. Kim, and D.-W. Kim, "Magnolol inhibits migration of vascular smooth muscle cells via cytoskeletal remodeling pathway to attenuate neointima formation," Experimental Cell Research, vol. 319, no. 20, pp. 3238-3250, 2013.

[56] R. Karki, C.-H. Park, and D.-W. Kim, "Extract of buckwheat sprouts scavenges oxidation and inhibits pro-inflammatory mediators in lipopolysaccharide-stimulated macrophages (RAW264.7)," Journal of Chinese Integrative Medicine, vol. 11, no. 4, pp. 246-252, 2013.

[57] R. Karki, N. Sahi, E.-R. Jeon, Y.-S. Park, and D.-W. Kim, "Chungtaejeon, a Korean fermented tea, scavenges oxidation and inhibits cytokine induced proliferation and migration of human aortic smooth muscle cells," Plant Foods for Human Nutrition, vol. 66, no. 1, pp. 27-33, 2011.

[58] R. Karki, E.-R. Jeon, and D.-W. Kim, "Nelumbo nucifera leaf extract inhibits neointimal hyperplasia through modulation of smooth muscle cell proliferation and migration," Nutrition, vol. 29, no. 1, pp. 268-275, 2013.

[59] R. Karki, O.-M. Ho, and D.-W. Kim, "Magnolol attenuates neointima formation by inducing cell cycle arrest via inhibition of ERK1/2 and NF- $\kappa \mathrm{B}$ activation in vascular smooth muscle cells," Biochimica et Biophysica Acta, vol. 1830, no. 3, pp. 26192628, 2013.

[60] G. Taraboletti, S. D’Ascenzo, P. Borsotti, R. Giavazzi, A. Pavan, and V. Dolo, "Shedding of the matrix metalloproteinases MMP2, MMP-9, and MT1-MMP as membrane vesicle-associated components by endothelial cells," The American Journal of Pathology, vol. 160, no. 2, pp. 673-680, 2002.

[61] H. C. Anderson, "Matrix vesicles and calcification," Current Rheumatology Reports, vol. 5, no. 3, pp. 222-226, 2003.

[62] R. Lacroix, F. Sabatier, A. Mialhe et al., "Activation of plasminogen into plasmin at the surface of endothelial microparticles: a mechanism that modulates angiogenic properties of endothelial progenitor cells in vitro," Blood, vol. 110, no. 7, pp. 2432-2439, 2007. 
[63] O. Morel, N. Morel, B. Hugel et al., "The significance of circulating microparticles in physiology, inflamatory and thrombotic diseases," La Revue de Médecine Interne, vol. 26, no. 10, pp. 791801, 2005 (French).

[64] A. H. M. Moons, M. Levi, and R. J. G. Peters, "Tissue factor and coronary artery disease," Cardiovascular Research, vol. 53, no. 2, pp. 313-325, 2002.

[65] J. Steffel, M. Hermann, H. Greutert et al., "Celecoxib decreases endothelial tissue factor expression through inhibition of c-Jun terminal NH2 kinase phosphorylation," Circulation, vol. 111, no. 13, pp. 1685-1689, 2005.

[66] E. Napoleone, A. Di Santo, and R. Lorenzet, "Monocytes upregulate endothelial cell expression of tissue factor: a role for cellcell contact and cross-talk," Blood, vol. 89, no. 2, pp. 541-549, 1997.

[67] M. Mesri and D. C. Altieri, "Leukocyte microparticles stimulate endothelial cell cytokine release and tissue factor induction in a JNK1 signaling pathway," Journal of Biological Chemistry, vol. 274, no. 33, pp. 23111-23118, 1999.

[68] A. Tesse, M. C. Martínez, B. Hugel et al., "Upregulation of proinflammatory proteins through NF- $\kappa$ B pathway by shed membrane microparticles results in vascular hyporeactivity," Arteriosclerosis, Thrombosis, and Vascular Biology, vol. 25, no. 12, pp. 2522-2527, 2005.

[69] A. Scanu, N. Molnarfi, K. J. Brandt, L. Gruaz, J.-M. Dayer, and D. Burger, "Stimulated T cells generate microparticles, which mimic cellular contact activation of human monocytes: differential regulation of pro- and anti-inflammatory cytokine production by high-density lipoproteins," Journal of Leukocyte Biology, vol. 83, no. 4, pp. 921-927, 2008.

[70] M. H. Gräler and E. J. Goetzl, "Lysophospholipids and their G protein-coupled receptors in inflammation and immunity," Biochimica et Biophysica Acta, vol. 1582, no. 1-3, pp. 168-174, 2002.

[71] A. S. Leroyer, F. Anfosso, R. Lacroix et al., "Endothelialderived microparticles: biological conveyors at the crossroad of inflammation, thrombosis and angiogenesis," Thrombosis and Haemostasis, vol. 104, no. 3, pp. 456-463, 2010.

[72] S. Simoncini, M.-S. Njock, S. Robert et al., "Trail/Apo2L mediates the release of procoagulant endothelial microparticles induced by thrombin in vitro: a potential mechanism linking inflammation and coagulation," Circulation Research, vol. 104, no. 8, pp. 943-951, 2009.

[73] F. Meziani, A. Tesse, E. David et al., "Shed membrane particles from preeclamptic women generate vascular wall inflammation and blunt vascular contractility," The American Journal of Pathology, vol. 169, no. 4, pp. 1473-1483, 2006.

[74] M. Mesri and D. C. Altieri, "Endothelial cell activation by leukocyte microparticles," Journal of Immunology, vol. 161, no. 8, pp. 4382-4387, 1998.

[75] S. H. van Ierssel, E. M. van Craenenbroeck, V. M. Conraads et al., "Flow cytometric detection of endothelial microparticles (EMP): effects of centrifugation and storage alter with the phenotype studied," Thrombosis Research, vol. 125, no. 4, pp. 332-339, 2010.

[76] P. J. A. Yong, C. H. Koh, and W. S. N. Shim, "Endothelial microparticles: missing link in endothelial dysfunction?" European Journal of Preventive Cardiology, vol. 20, no. 3, pp. 496-512, 2013.

[77] R. Soleti, T. Benameur, C. Porro, M. A. Panaro, R. Andriantsitohaina, and M. C. Martínez, "Microparticles harboring Sonic
Hedgehog promote angiogenesis through the upregulation of adhesion proteins and proangiogenic factors," Carcinogenesis, vol. 30, no. 4, pp. 580-588, 2009.

[78] P. E. Rautou, A. S. Leroyer, B. Ramkhelawon et al., "Microparticles from human atherosclerotic plaques promote endothelial ICAM-1-dependent monocyte adhesion and transendothelial migration," Circulation Research, vol. 108, no. 3, pp. 335-343, 2011.

[79] K. D. O’Brien, T. O. McDonald, A. Chait, M. D. Allen, and C. E. Alpers, "Neovascular expression of E-selectin, intercellular adhesion molecule-1, and vascular cell adhesion molecule-1 in human atherosclerosis and their relation to intimal leukocyte content," Circulation, vol. 93, no. 4, pp. 672-682, 1996.

[80] R. Virmani, F. D. Kolodgie, A. P. Burke et al., "Atherosclerotic plaque progression and vulnerability to rupture: angiogenesis as a source of intraplaque hemorrhage," Arteriosclerosis, Thrombosis, and Vascular Biology, vol. 25, no. 10, pp. 2054-2061, 2005.

[81] P. R. Moreno, K.-R. Purushothaman, M. Sirol, A. P. Levy, and V. Fuster, "Neovascularization in human atherosclerosis," Circulation, vol. 113, no. 18, pp. 2245-2252, 2006.

[82] M. Jayachandran, R. D. Litwiller, W. G. Owen, and V. M. Miller, "Circulating microparticles and endogenous estrogen in newly menopausal women," Climacteric, vol. 12, no. 2, pp. 177-184, 2009.

[83] N. Figueroa-Vega, C. Moreno-Frías, and J. M. Malacara, "Alterations in adhesion molecules, pro-inflammatory cytokines and cell-derived microparticles contribute to intima-media thickness and symptoms in postmenopausal women," PLoS ONE, vol. 10, no. 5, Article ID e0120990, 2015.

[84] M. Jayachandran, R. D. Litwiller, B. D. Lahr et al., "Alterations in platelet function and cell-derived microvesicles in recently menopausal women: relationship to metabolic syndrome and atherogenic risk," Journal of Cardiovascular Translational Research, vol. 4, no. 6, pp. 811-822, 2011.

[85] M.-V. Noci, R. Ramírez, M. Lluch, M. Rodríguez, and J. Carracedo, "Changes in endothelial microparticles and endothelial progenitor cells in obese patients in response to surgical stress," The Journal of Bone \& Joint Surgery -American Volume, vol. 97, no. 5, pp. 353-358, 2015.

[86] L. F. Heinrich, D. K. Andersen, M. E. Cleasby, and C. Lawson, "Long-term high fat feeding of rats results in increased numbers of circulating microvesicles with pro-inflammatory effects on endothelial cells," British Journal of Nutrition, vol. 113, no. 11, pp. 1704-1711, 2015. 


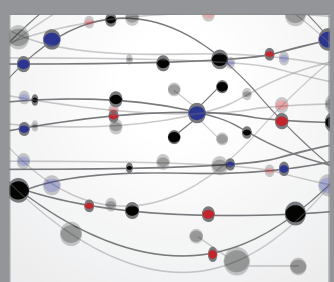

The Scientific World Journal
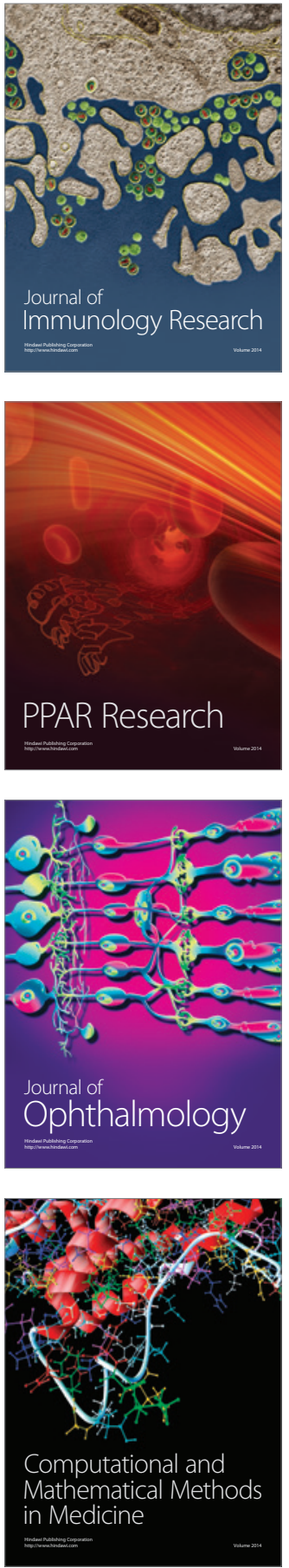

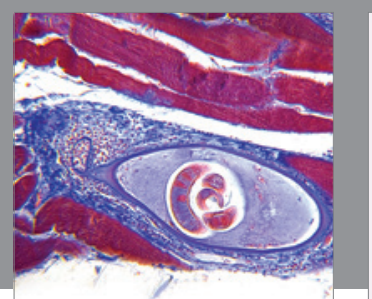

Gastroenterology Research and Practice

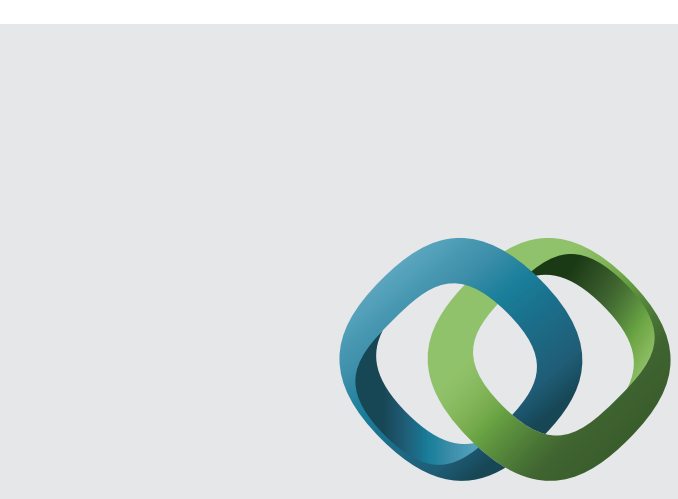

\section{Hindawi}

Submit your manuscripts at

http://www.hindawi.com
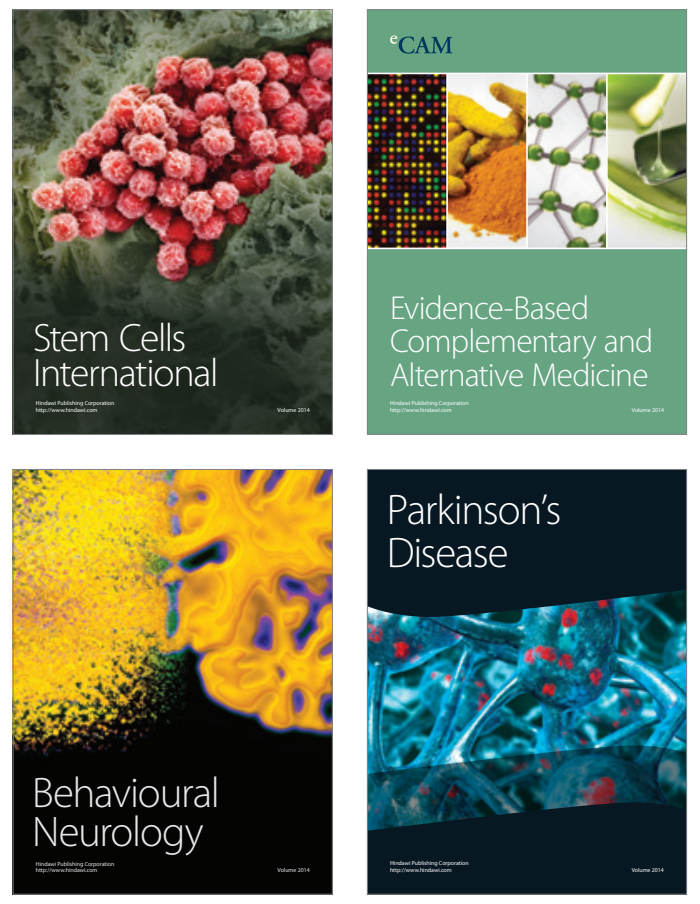
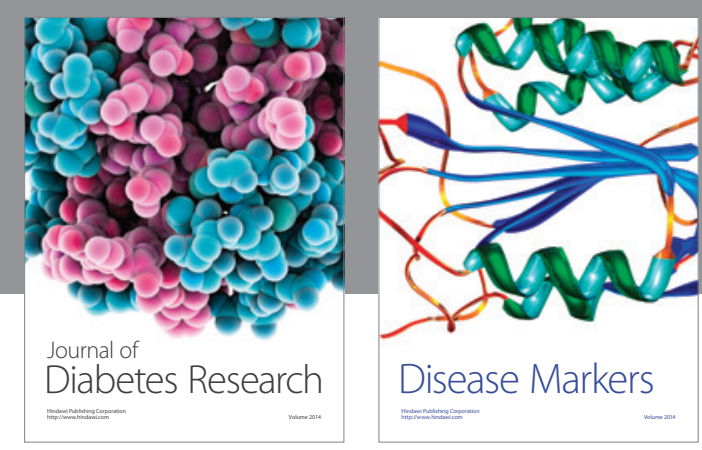

Disease Markers
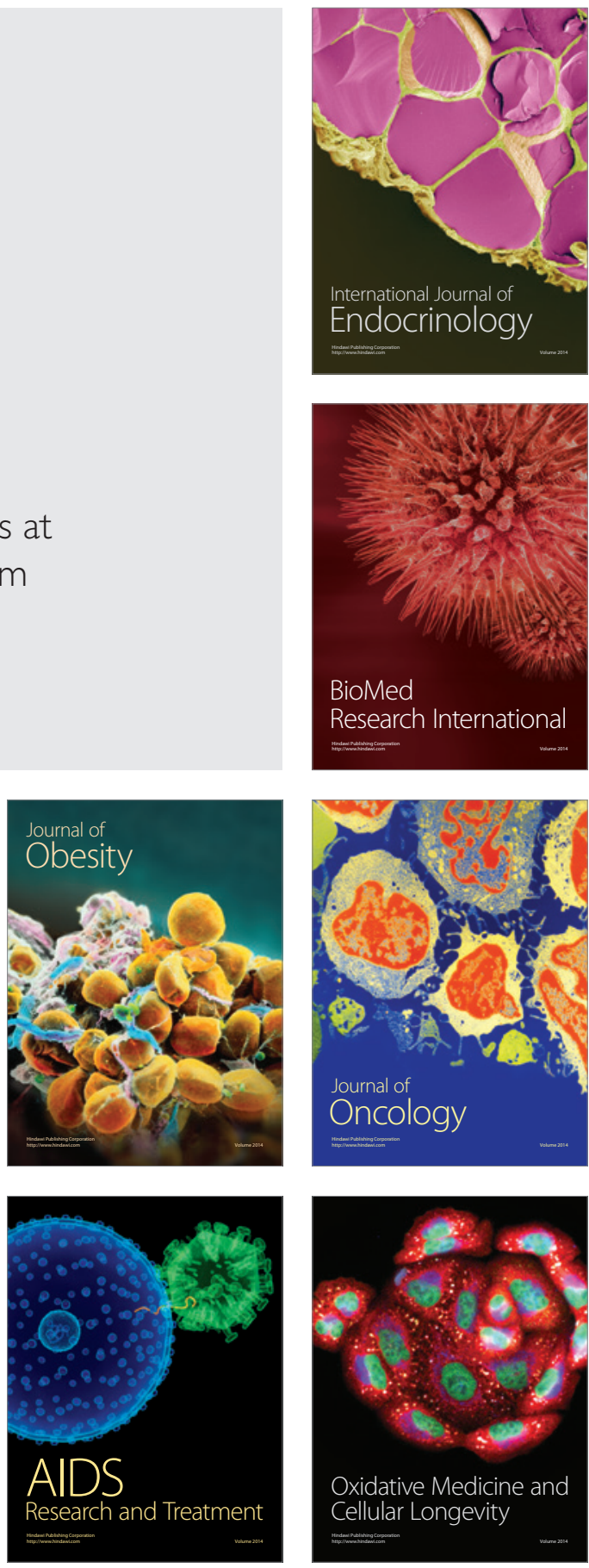